\title{
Shear stress effect on endothelial nitric oxide synthase in cultured human umbilical vein endothelial cells
}

\author{
Qiuying Gu${ }^{1}$, Dean O. Smith ${ }^{2}$, Karlene A. Hoo $^{1^{*}}$ \\ ${ }^{1}$ Departments of Chemical Engineering, Texas Tech University, Lubbock, USA \\ ${ }^{2}$ Departments of Biological Sciences, Texas Tech University, Lubbock, USA \\ Email: "karlene.hoo@ttu.edu.
}

Received 16 August 2013; revised 12 September 2013; accepted 18 September 2013

Copyright (c) 2013 Qiuying Gu et al. This is an open access article distributed under the Creative Commons Attribution License, which permits unrestricted use, distribution, and reproduction in any medium, provided the original work is properly cited.

\begin{abstract}
Background: Low shear stress caused by disturbed or turbulent flow at arterial branch points is known to associate with atherosclerosis. However, shear stress at the venous valve location and its association with deep vein thrombosis are less understood due to the complex and poorly understood bi-directional flow in the valve pocket region. We investigated how venous endothelial cells respond to flow shear stress around the venous valve region using a novel in vitro system that mimics venous flow. Results: Human umbilical vein EA hy. 926 cells were cultured on a flexible silastic membrane that mimicked venous tissue. Confluent cells were exposed to sinusoidal uni- and bi-directional pulsatile shear stress $\left(0.1\right.$ to $\left.1 \mathrm{dyne} / \mathrm{cm}^{2}\right)$ for up to $6 \mathrm{~h}$. Western-blot analyses indicated that endothelial nitric oxide (eNOS) expression levels decreased regardless of all tested flow patterns, stress magnitude, and shearing time. In contrast, the expression levels of inhibitor of $\boldsymbol{\kappa} B$ (kappa B) and $\alpha$ (alpha)-tubulin were unaffected by the shear stress. Conclusions: Our results indicate that shear stress causes a decrease specifically in eNOS expression, suggesting that it may play a significant role in regulating inflammation related protein expression in endothelial cells.
\end{abstract}

Keywords: Shear Stress; Endothelial Cells; Endothelial Nitric Oxide Synthase; Inflammation; Thrombosis

\section{INTRODUCTION}

The endothelium is a metabolically active cell layer involved in many homeostatic processes, including leukocyte adhesion and the maintenance of vascular tone by its response to physiological stimuli, because of its unique location at the interface of blood flow and vessel wall [1].

*Corresponding author.
Inflammation of the endothelium is atherogenic and considered to play a major role in the pathology of atherosclerosis [2,3]. Different types of hemodynamic shear stress can lead to various endothelial phenotypes exhibiting inflammatory responses, many of which reflect alternations in certain contamination protein expression levels $[4,5]$. In particular, the involvement of endothelial nitric oxide synthase (eNOS) in inflammatory processes has been widely studied and shown to respond to shear stress by secreting the vasodilator nitric oxide (NO) [6]. Recent studies have reinforced the importance of this protective effect to vascular health $[7,8]$. Moreover, nuclear factor- $\kappa \mathrm{B}$ (NF- $\kappa \mathrm{B}$ ), which is modulated by $\mathrm{I} \kappa \mathrm{B}$ (inhibitor of $\kappa \mathrm{B}$ ), has high activity in the regions with oscillatory and turbulent flows [9]. In arteries with impaired flow, NF- $\kappa$ B dependent transcription regulates vasoprotection, mainly by decreased NO production and increased pro-inflammatory mediator expression [9].

These endothelium protein regulatory responses to different types of steady and unsteady shear stress have been analyzed mainly in atherosclerotic lesions [5]. However, the possible impairment of valvular sinus endothelium functions due to venous valve failure and venous reflux has received little attention in the studies of thrombosis risk $[10,11]$. The deep venous valvular sinus is known to be a unique micro-environment exposed to cyclic hypoxia, stasis, and hyper-coagulability with pulsatile, unsteady, bi-directional flow because of the flexible elastic structure of venous valve leaflets and their motions [12]. The possible connection between the flow patterns in the venous valve pockets and thrombus formation has been elucidated in vivo, where the counterrotating secondary vortex fluid circulating with extremely low velocities can create a very low shear field that could promote the aggregation of blood cells [13].

Controlled in vitro studies of shear stress in venous tissue have been hindered by the difficulties of constructing an experimental apparatus that mimics the elastic in 
vivo micro-environment. We have overcome this difficulty by using a novel silastic membrane and have successfully monitored eNOS and $\mathrm{I} \kappa \mathrm{B}$ expression levels in response to shear stresses with varying strength and time scales.

\section{MATERIALS AND METHODS}

\subsection{Flexible Membrane Preparation}

To mimic the flexible venous valve structure, flexible membranes were made from Dow Corning Sylgard 184 silicone elastomer according to the manufacturer's instructions. This silastic membrane surface is highly hydrophobic, so to facilitate cell attachment, it was coated with a $0.3 \%$ rat tail collagen solution in HEPES-buffered (1.75 mmol/L) modified Eagle’s medium [14].

\subsection{Cell Culture}

EA hy. 926, a hybridoma cell line obtained by the hybridization of human umbilical vein endothelial cells (HUVECs) with the A549/8 human lung carcinoma cell line (passages 2 to 7), were used because they preserve many features of HUVECs as a preferable homogeneous experimental model of angiogenesis in vitro because it demonstrates highly differentiated functions of human vascular endothelium, while providing the advantage of immortality, stability through passage number and the reproducibility of results [15]. Cells were cultured initially in tissue-culture flasks at $37^{\circ} \mathrm{C}$ with $5 \% \mathrm{CO}_{2}$ in Dulbecco's modified Eagle's medium (DMEM), supplemented with 10\% FBS and 1\% penicillin and streptomycin [16]. At confluence, they were harvested using $0.25 \%$ trypsin and re-plated on the silastic membranes (4 $\mathrm{mL}$ with $5 \times 10^{5}$ cells $/ \mathrm{mL}$ ). A square retaining ring was used to hold the solution onto the membrane. It took $12 \mathrm{~h}$ for the cells to adhere fully and $48 \mathrm{~h}$ to become a confluent monolayer. The retaining ring was removed before the shearing experiment.

\subsection{Shearing Stress}

Silastic membranes holding the confluent monolayer of cells were transferred to an individual parallel plate flow chamber system (Figure 1). Culture medium was circulated using a peristaltic pump connected to a function generator that determined flow direction via sinusoidal waveforms. The period of the pulsed sine wave flow mimics the in vivo $3 \mathrm{~s}$ duration of one valve cycle [17].

Furthermore, when generating the bi-directional flow, in order to imitate the valve operating cycle in vivo [17], shearing flow was provided for $2 / 3$ of the sinusoid period in the forward direction and $1 / 3$ in the reverse direction. The chamber and medium were placed in a $37^{\circ} \mathrm{C}$ incubator with $5 \% \mathrm{CO}_{2}$ for 3 to $6 \mathrm{~h}$ of shearing.

At steady-state channel flow in the chamber, the shear

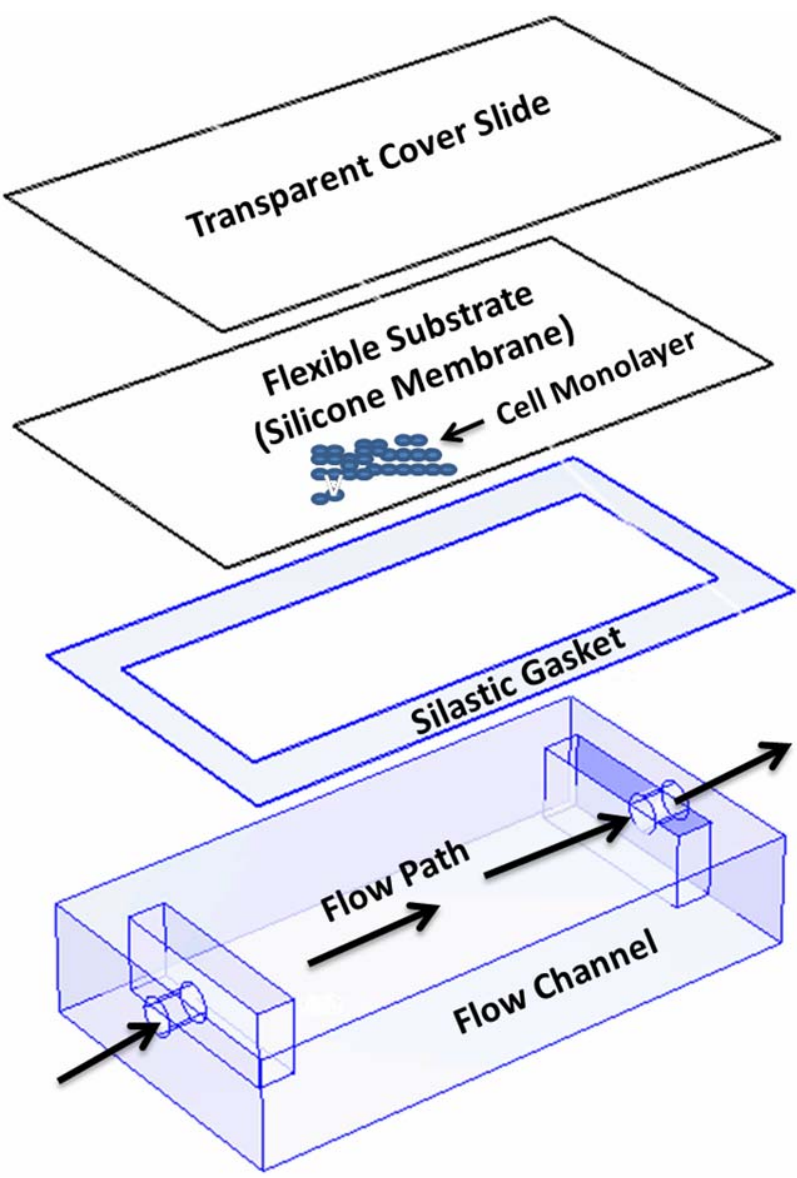

Figure 1. Schematic diagram of the parallel plate flow chamber, including the slide attached to the silicone membrane occupied with confluent endothelium monolayer, the rectangular silastic gasket and the base plate. The apparatus is hold together by a steel binder, and a gap width of $0.25 \mathrm{~mm}$ was formed between the membrane and the plate surface.

stress, $\tau$, is calculated as:

$$
\tau=\frac{6 \mu Q}{h^{2} w}
$$

where $\mu$ is the dynamic medium viscosity (0.008 poise), $Q$ is the fluid flow rate (mL/s), $h$ is the gasket thickness $(0.25 \mathrm{~mm})$, and $w$ is the width of the chamber $(25 \mathrm{~mm})$. Different shearing stresses can be adjusted by changing the pump's flow rate.

\subsection{Western Blots}

At the conclusion of a shearing experiment, the membrane surface was washed with ice-cold phosphate-buffered saline, and the cells were harvested with a rubber scraper. After centrifugation, the cells were suspended in Ripa lysis buffer and proteins were denatured at $95^{\circ} \mathrm{C}$ for 5 min. The protein samples (30 micrograms) were loaded on a $10 \%$ sodium dodecyl sulfate polyacrylamide gel and electrophoresed at $100 \mathrm{~V}$ for $1.5 \mathrm{~h}$ and then transferred 
onto a polyvinylidene fluoride membrane at $45 \mathrm{~V}$ for 2.5 h. After blocking the membrane with $5 \%$ milk solution at room temperature, they were exposed to antibodies against eNOS (BD Biosciences, Cat.\# 610296, 1:1000), I $\kappa$ B (Cell Signaling, Cat.\# 9242, 1:1000), and $\alpha$-tubulin (Santa Cruz Biotechnology, Cat.\# sc-12462-R, 1:1000).

The HRP-linked secondary antibodies (Cell-Signaling, Cat.\# 7074, 1:1000), were detected through chemiluminescence (Immun-Star HRP Substrate, Bio-Rad, cat. \# 170-5041) exposed onto X-ray film. Two independent observers estimated the intensity of each band by eye, using a relative score ranging from 1 to 10 ; the average values were used for subsequent statistical analyses.

\section{RESULTS}

As shear stress increased from very low control levels (0.1 dyne $/ \mathrm{cm}^{2}$ ), eNOS expression decreased (Figure 2). Indeed, regardless of flow direction (forward or forward/ reverse), time ( 3 or $6 \mathrm{~h}$ ), or stress amplitude ( 0.5 or 1.0 dyne/ $\left.\mathrm{cm}^{2}\right)$, eNOS expression was significantly lower than the very low level control (one way ANOVA, $\mathrm{F}_{9,49}=$ $5.21, \mathrm{P}<0.01)$. In contrast, neither I $\kappa \mathrm{B}$ nor $\alpha$-tubulin expression varied significantly with shear stress (oneway ANOVA, $\mathrm{F}_{9,30}=1.19, \mathrm{P}>0.05$, and $\mathrm{F}_{4,40}=1.00, \mathrm{P}>$ 0.05 , respectively). Thus, the effects of shear stress on eNOS are not non-specific.

The dependence of eNOS expression on stress ampli- tude, shear flow direction, and shearing time was examined further. There are no significant differences in expression levels as stress magnitude increases from 0.5 to $1 \mathrm{dyne} / \mathrm{cm}^{2}$ for samples exposed to either forward shearing flow for $3 \mathrm{~h}\left(\mathrm{t}_{\mathrm{df}=12}=0.56, \mathrm{P}>0.05\right)$ or bi-directional shearing flow for $3 \mathrm{~h}\left(\mathrm{t}_{\mathrm{df}=12}=1.70, \mathrm{P}>0.05\right)$ or $6 \mathrm{~h}\left(\mathrm{t}_{\mathrm{df}=12}\right.$ $=1.22, \mathrm{P}>0.05)$. Anomalously, eNOS expression decreased by $54 \%$ between samples exposed to $0.5 \mathrm{dyne} / \mathrm{cm}^{2}$ versus 1 dyne $/ \mathrm{cm}^{2}$ for forward shearing flow at $6 \mathrm{~h}\left(\mathrm{t}_{\mathrm{df}=12}\right.$ $=2.28, \mathrm{P}<0.05)$. No significant differences in eNOS expression between forward and bi-directional flow were observed at both $3 \mathrm{~h}$ and $6 \mathrm{~h}$ of flow exposure. However, for forward flow with magnitude $1 \mathrm{dyne} / \mathrm{cm}^{2}$, eNOS expression decreased $77 \%$ as exposure time change from 3 $\mathrm{h}$ to $6 \mathrm{~h}\left(\mathrm{t}_{\mathrm{df}=12}=4.51, \mathrm{P}<0.01\right)$. For bi-directional flow with stress magnitude of 1 dyne/cm ${ }^{2}$, eNOS expression decreased $43 \%$ between samples taken at $3 \mathrm{~h}$ and $6 \mathrm{~h}$ $\left(\mathrm{t}_{\mathrm{df}=12}=2.18, \mathrm{P}<0.05\right)$.

\section{DISCUSSION}

Under experimental conditions mimicking the flexible venous wall, we demonstrate that shear stress decreases eNOS expression. This occurs in a specific manner, for shear stress does not alter either $\mathrm{I} \kappa \mathrm{B}$ or $\alpha$-tubulin. Furthermore, the decrease in eNOS expression increases as the duration of shearing becomes longer, indicating the presence of a pro-inflammatory environment [18].

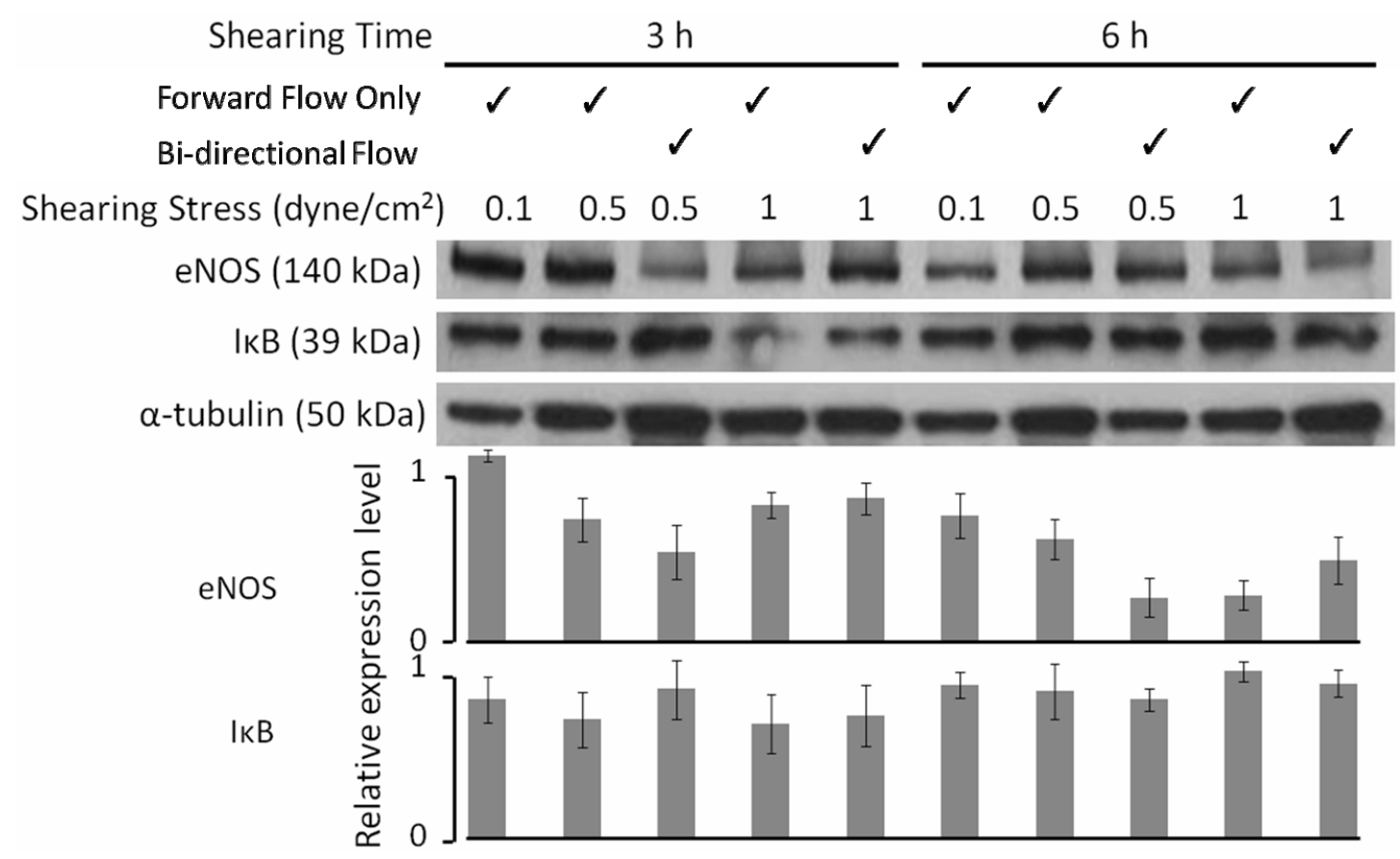

Figure 2. Western blot analysis of proteins eNOS, $\alpha$-tublin and I $\kappa \mathrm{B}$ obtained from endothelium either maintained under weak flow conditions (forward flow with stress magnitude $0.1 \mathrm{dyne} / \mathrm{cm}^{2}$ ) or exposed to pulsatile, uni-/bi-directional flow with magnitude from 0 to $1 \mathrm{dyne} / \mathrm{cm}^{2}$ for variable time intervals. Data from seven separate repetitions are expressed as mean \pm S.E. All eNOS levels are significantly less $(\mathrm{P}<0.05)$ than the value at 0.1 dyne $/ \mathrm{cm}^{2}$ for $3 \mathrm{~h}$ (far left bar). There are no significant differences $(\mathrm{P}>0.05)$ within any of the $\mathrm{I} \kappa \mathrm{B}$ or the $\alpha$-tubulin data. 
This may appear to contradict with some evidence stating that shear stress upregulates eNOS in HUVECs [5, 19]. However, the contradictory results were obtained under different shearing environments. Specifically, the stress applied in our work (on the order of $1 \mathrm{dyn} / \mathrm{cm}^{2}$ ) was an order of magnitude lower than the levels (on the order of $5-25 \mathrm{dyn} / \mathrm{cm}^{2}$ ) reported in previous works [5, 19]. This was specifically chosen to better mimic the venous flow representing path physiologically relevant stimuli in inflammation.

Selective regulation of eNOS expression in cultured HUVECs exposed to shear stress with different magnitude, shear flow direction and exposure time may account for the different effects of shear stress properties. First, Shear stress initiates the decrease of eNOS expression compared to control experiments conducted under near static flow regardless of flow direction, time, or stress amplitude. This indicates that our shearing conditions are pro inflammatory [18], which is also consistent with the conclusion that shear stress plays an important role in regulation the inflammatory process. Second, monitoring the gene expression in our shearing condition can help to evaluate how eNOS expression is regulated by shear stress in environment after inflammation was initiated. We reported here that eNOS expression under 1 dyne $/ \mathrm{cm}^{2}$ shear stress decreases with exposure term, confirming the temporal effect of low magnitude shear stress on inflammatory genes expression. This finding supports our hypothesis that long term application of low magnitude shear stress tends to generate a pro-inflammatory environment, which also may help explain thrombosis in venous valve regions with low blood flow.

$\mathrm{I} \kappa \mathrm{B}$ protein is tightly related to the activity of transcription factor NF- $\kappa \mathrm{B}$, which is predicted to mediate inflammatory gene expression in response to shear stress $[9,20]$. Under the shearing conditions adopted in this study, however, I $\kappa \mathrm{B}$ expression level was not significantly affected by shear stress. This result may indicate that the shearing conditions studied are not the ones responsible for generating significant $\mathrm{I} \kappa \mathrm{B}$ changes, which implies that the combinations of flow patterns and magnitudes used are not related to the onset of severe inflammation associated with increased $\mathrm{I} \kappa \mathrm{B}$ expression.

Our results obtained from HUVECs contrast with the arterial endothelial cells, where increased shear stress increases eNOS expression [2]. Conversely, arterial branch points and curved regions having disturbed or turbulent flow (and, therefore, low shear stresses) reduce eNOS expression, thus disrupting nitric oxide-dependent atheroprotection and stimulating atherosclerosis [21]. Shear stress at the level present in arteries $\left(>15\right.$ dyne $\left./ \mathrm{cm}^{2}\right)$ induces atheroprotective gene expression, while low stress $\left(<4\right.$ dyne/ $\left.\mathrm{cm}^{2}\right)$ stimulates atherogenic endothelial phenotypes [22]. Indeed, one of the earliest biochemical changes preceding endothelial dysfunction is decreased expression of an important vasodilator, nitric oxide (NO), due to decreased eNOS expression [23].

By extension, we hypothesize that venous valve regions, characterized by complex pulsatile flow, also may relate closely to endothelial dysfunction and thrombosis in venous systems. As our study shows, this presumably also involves reduced eNOS and, therefore, NO production.

One deficiency of the experimental model used in our work is that the novel flow chamber could not withstand the high stress conditions that previous works have used to simulate aerterial flows, so therefore we could not generate results in this flow region for comparison to these previous studies. On the other hand, hybridoma cell line EA hy. 926 has been shown to preserve many features of HUVECs as a preferable homogeneous experimental model of angiogenesis in vitro because it demonstrates highly differentiated functions of human vascular endothelium, while providing the advantage of immortality, stability through passage number and the reproducibility of results [15]. Our results shown here also indicate that EA hy. 926 can be safely used as a model of evaluating eNOS expression in ECs under low shear stress environment.

\section{CONCLUSION}

In conclusion, our findings provide further evidence that shearing stress plays a significant role in endothelial functions related to venous inflammation. As discussed, inflammatory protein expression in endothelium can be, at least temporarily, differentially regulated by shearing stresses of different magnitudes and flow patterns. Shear stress initiates the decrease of eNOS expression compared to control experiments conducted under near static flow. These observations shed further light on the inflammatory response in regions at high risk of thrombosis. Further studies evaluating other potentially important factors prone to thrombosis would contribute to identifying the specific blood flow pattern correlated to thrombosis onset. Such studies hopefully will provide insights into finding the pro-thrombosis factors that, rather than flow pattern, other flow-related conditions may play a more important role in venous thrombosis. In addition, this may lead to a more comprehensive understanding of the mechanisms explaining the initiation of thrombosis. A complex interplay of mechanical force factors remains to be investigated.

\section{REFERENCES}

[1] Aird, W. (2005) Spatial and temporal dynamics of the endothelium. Journal of Thrombosis and Haemostasis, 3, 1392-1406. 
http://dx.doi.org/10.1111/j.1538-7836.2005.01328.x

[2] Chatzizisis, Y.S., Coskun, A.U., Jonas, M., Edelman, E.R., Feldman, C.L. and Stone, P.H. (2007) Role of endothelial shear stress in the natural history of coronary atherosclerosis and vascular remodeling: Molecular, cellular, and vascular behavior. Journal of the American College of Cardiology, 49, 2379-2393.

http://dx.doi.org/10.1016/j.jacc.2007.02.059

[3] Aird, W.C. (2007) Phenotypic heterogeneity of the endothelium. Circulation research, 100, 174-190. http://dx.doi.org/10.1161/01.RES.0000255690.03436.ae

[4] Nagel, T., Resnick, N., Atkinson, W.J., Dewey Jr., C.F. and Gimbrone Jr., M.A. (1994) Shear stress selectively upregulates intercellular adhesion molecule-1 expression in cultured human vascular endothelial cells. Journal of Clinical Investigation, 94, 885. http://dx.doi.org/10.1172/JCI117410

[5] Barakat, A.I. and Lieu, D.K. (2003) Differential responsiveness of vascular endothelial cells to different types of fluid mechanical shear stress. Cell Biochemistry and Biophysics, 38, 323-343. http://dx.doi.org/10.1385/CBB:38:3:323

[6] Cirino, G., Fiorucci, S. and Sessa, W.C. (2003) Endothelial nitric oxide synthase: The Cinderella of inflammation? Trends in Pharmacological Sciences, 24, 91-95. http://dx.doi.org/10.1016/S0165-6147(02)00049-4

[7] Förstermann, U. and Li, H. (2011) Therapeutic effect of enhancing endothelial nitric oxide synthase (eNOS) expression and preventing eNOS uncoupling. British Journal of Pharmacology, 164, 213-223. http://dx.doi.org/10.1111/j.1476-5381.2010.01196.x

[8] Zhang, Y., Janssens, S.P., Wingler, K., Schmidt, H.H.H. W. and Moens, A.L. (2011) Modulating endothelial nitric oxide synthase: A new cardiovascular therapeutic strategy. American Journal of Physiology-Heart and Circulatory Physiology, 301, H634-H646. http://dx.doi.org/10.1152/ajpheart.01315.2010

[9] Cunningham, K.S. and Gotlieb, A.I. (2004) The role of shear stress in the pathogenesis of atherosclerosis. Laboratory Investigation, 85, 9-23. http://dx.doi.org/10.1038/labinvest.3700215

[10] Quyyumi, A.A. (1998) Endothelial function in health and disease: New insights into the genesis of cardiovascular disease. The American Journal of Medicine, 105, 32S-39S. http://dx.doi.org/10.1016/S0002-9343(98)00209-5

[11] Nicolaides, A. (2000) Investigation of chronic venous insufficiency: A consensus statement. Circulation, 102, e126-e163. http://dx.doi.org/10.1161/01.CIR.102.20.e126

[12] Hamer, J., Malone, P. and Silver, I. (1981) The PO2 in venous valve pockets: Its possible bearing on thrombogenesis. British Journal of Surgery, 68, 166-170. http://dx.doi.org/10.1002/bjs.1800680308
[13] Karino, T. and Motomiya, M. (1984) Flow through a venous valve and its implication for thrombus formation. Thrombosis Research, 36, 245-257. http://dx.doi.org/10.1016/0049-3848(84)90224-X

[14] Simpson, D., Majeski, M., Borg, T. and Terracio, L. (1999) Regulation of cardiac myocyte protein turnover and myofibrillar structure in vitro by specific directions of stretch. Circulation Research, 85, e59. http://dx.doi.org/10.1161/01.RES.85.10.e59

[15] Edgell, C.J.S., Haizlip, J.E. and Bagnell, C.R. (1990) Endothelium specific Weibel-Palade bodies in a continuous human cell line, EA. hy926. In Vitro Cellular \& Developmental Biology-Plant, 26, 1167-1172. http://dx.doi.org/10.1007/BF02623694

[16] Edgell, C., McDonald, C.C. and Graham, J.B. (1983) Permanent cell line expressing human factor VIII-related antigen established by hybridization. Proceedings of the National Academy of Sciences, 80, 3734. http://dx.doi.org/10.1073/pnas.80.12.3734

[17] Lurie, F., Kistner, R.L., Eklof, B. and Kessler, D. (2003) Mechanism of venous valve closure and role of the valve in circulation: A new concept. Journal of Vascular Surgery, 38, 955-961. http://dx.doi.org/10.1016/S0741-5214(03)00711-0

[18] Guzik, T., Korbut, R. and Adamek-Guzik, T. (2003) Nitric oxide and superoxide in inflammation. Journal of Physiology and Pharmacology, 54, 469-487.

[19] Harrison, D.G., Sayegh, H., Ohara, Y., Inoue, N. and Venema, R.C. (1996) Regulation of expression of the endothelial cell nitric oxide synthase. Clinical and Experimental Pharmacology and Physiology, 23, 251-255. http://dx.doi.org/10.1111/j.1440-1681.1996.tb02606.x

[20] Li, Y., Haga, J. and Chien, S. (2005) Molecular basis of the effects of shear stress on vascular endothelial cells. Journal of Biomechanics, 38, 1949-1971. http://dx.doi.org/10.1016/j.jbiomech.2004.09.030

[21] Papafaklis, M.I., Koskinas, K.C., Chatzizisis, Y.S., Stone, P.H. and Feldman, C.L. (2010) In vivo assessment of the natural history of coronary atherosclerosis: vascular remodeling and endothelial shear stress determine the complexity of atherosclerotic disease progression. Current Opinion in Cardiology, 25, 627. http://dx.doi.org/10.1097/HCO.0b013e32833f0236

[22] Malek, A.M., Alper, S.L. and Izumo, S. (1999) Hemodynamic shear stress and its role in atherosclerosis. The Journal of the American Medical Association, 282, 20352042. http://dx.doi.org/10.1001/jama.282.21.2035

[23] Casas, J.P., Bautista, L.E., Humphries, S.E. and Hingorani, A.D. (2004) Endothelial nitric oxide synthase genotype and ischemic heart disease. Circulation, 109, 13591365.

http://dx.doi.org/10.1161/01.CIR.0000121357.76910.A3 